\title{
On the plate-like and layer-like response of slab foundations to ground-borne vibration
}

\author{
G. Sanitate, J.P. Talbot \\ Department of Engineering, University of Cambridge, Trumpington Street, Cambridge CB2 1PZ, UK
}

\begin{abstract}
The increasing urban population is leading to the exploitation of building sites, close to surface or underground railways, with considerable levels of ground-borne vibration. An important design consideration regards the levels of perceptible vibration and/or re-radiated noise in the completed buildings. A fundamental question concerns to what extent the mass and stiffness of a building foundation influences these levels. This paper explores this question in relation to a concrete slab foundation.

Previous research has explored the influence of the coupling between a thin, flexural plate and an elastic half-space on the free-surface displacements arising from surface Rayleigh waves. Here, a numerical, wavebased approach is used to model the slab foundation as an elastic layer of finite thickness, overlying the half-space. The latter is subjected to incident waves in the form of Rayleigh, P- and SV-waves. It is found that thin-plate theory alone is insufficient for modelling the slab over the full frequency range of interest, and that the assumed soil-slab boundary condition plays a significant role. Design plots are presented in order to summarise the influence of the salient dimensionless parameters, and to help guide the design of a slab foundation to achieve a specific reduction in ground vibration level.
\end{abstract}

Keywords: foundation, ground-borne vibration, soil-structure interaction, railway

\section{Introduction}

Ground-borne vibration generated by railways is an increasing concern for building designers. Vibration generated at the wheel-rail interface propagates through the track and supporting structures, into the un-

4 derlying ground, from where it may propagate to the foundations of nearby buildings. Depending on the mass, stiffness and damping distribution within a building and its foundation, the resulting levels of per-

Email addresses: gs528@cam.ac.uk (G. Sanitate), jpt1000@cam.ac.uk (J.P. Talbot) 
ceptible vibration and re-radiated noise may lead to the annoyance of occupants or, in the case of specialist manufacturing or research facilities, the malfunctioning of sensitive equipment.

A number of strategies are available for mitigating ground-borne vibration in buildings, by addressing the source [1, 2], the transmission path [3, 4] or the receiver (building) [5]. One example of mitigation at the receiver is the introduction of a thick slab foundation to help suppress the vibration, primarily by stiffening the base of the building. This approach is usually employed for specialist manufacturing facilities, such as silicon wafer fabs [6], where particularly stringent vibration criteria must be met. It has also been suggested as a straightforward approach for commercial and residential buildings, since it may simply involve constructing a thicker slab than pure structural considerations dictate. However, the extent to which a slab influences the ground vibration field is not clear, and there is little or no guidance for designers wishing to achieve a specific reduction in ground vibration level.

The behaviour of a slab foundation resting on a soil deposit subject to ground-borne vibration represents a particular case of soil-structure interaction (SSI). Significant literature exists on the SSI associated with both flexible and rigid foundations [7, 8, 9, 10]. However, the main focus has been earthquake-related problems, which involve low frequencies and long wavelengths, and therefore allow the foundations to be treated as rigid. In the case of ground-borne vibration due to railways, the frequency range of interest extends to much higher frequencies, typically from approximately $25 \mathrm{~Hz}$ to $250 \mathrm{~Hz}$ [11], and therefore involves relatively short wavelengths that are comparable with the dimensions of a typical foundation. In this case, the flexibility of a foundation is one of the dominant factors governing its response.

In the context of ground-borne vibration, Auersch [12] considers the response of thin, flexural plates (representing the foundation slab) resting on an elastic half-space (the soil deposit) to surface Rayleigh wave excitation. Both finite and infinitely-long plates are considered, using a combined finite-element boundaryelement method, and a semi-analytical method in the frequency-wavenumber domain. Valuable results from a parametric study are presented that considers the influence of mass, stiffness and soil layering on the soilslab interaction and the extent to which a slab attenuates ground vibration levels. In particular, Auersch concludes that the slab thickness is the dominant parameter governing the level of attenuation, since this essentially governs the frequency above which attenuation occurs.

The work presented here begins by reproducing some of the results of Auersch, this time using the dynamic stiffness method (DSM) implemented within the Elastodynamics Toolbox [13, 14] in MATLAB [15]. The foundation slab is now modelled as an elastic layer, of infinite horizontal extent but finite thickness, 
overlying an elastic half-space. The assumption of infinite horizontal extent is unsuitable for modelling the detailed response of a particular foundation to a particular vibration source. In particular, it fails to account for any modal behaviour of the slab due to the absence of wave reflections from the slab boundary. However, these modes are often heavily damped, due to the radiation damping provided by the soil, and are of secondary interest to the fundamental, underlying SSI. Auersch demonstrates that, for the case of Rayleigh wave excitation and for a relaxed boundary condition at the soil-foundation interface, the SSI associated with a finite plate is approximated well by that of an infinitely long one. An infinite slab is therefore adopted here to investigate further some of the fundamental behaviour: to enable the identification of the salient non-dimensional groups; and to investigate the influence of the assumed boundary conditions at the soil-slab interface, as well as the effect of the plate-like assumption for the slab. The assumption of linear-elastic soil behaviour is justified on the basis of the low strain levels associated with ground-borne vibration, which usually lie within the elastic regime for most soils (see, for example, Connolly et al. [16]).

Having considered surface Rayleigh wave excitation, the case of a buried source is investigated by considering incident $\mathrm{P}$ - and SV-waves, before considering the overall implications of the study for foundation design.

\section{Overview of the problem}

Our interest is the particular SSI associated with a flexible, concrete slab foundation, that is, the influence of such a slab on the free-surface vibration field, expressed in terms of the ratio of the vibration amplitudes before and after the construction of the slab. As a first approximation, the slab may be assumed to be of infinite horizontal extent. This assumption is supported by Auersch, who demonstrates that, for the case of Rayleigh wave excitation, the SSI associated with a finite plate may be approximated by that of an infinitely long one. Here, the slab is treated as an elastic layer of thickness $h$ and infinite extent in both the $x$ and $y$ directions, with shear modulus $G_{c}$, Poisson's ratio $v_{c}$ and mass density $\rho_{c}$ (see Figure 11). The underlying soil is modelled as a homogeneous, isotropic, elastic half-space, with shear modulus $G_{s}$, Poisson's ratio $v_{s}$ and mass density $\rho_{s}$. Damping in both the slab and the soil is assumed to be hysteretic, as described by a frequency-independent loss factor in shear, with no associated damping in dilatation (see Hunt [17]). Both surface and body, plane-wave excitation is considered: a Rayleigh wave travelling in the $\mathrm{x}$-direction with speed $V_{R}$, and either an incident P- or SV-wave travelling respectively with speed $V_{P}$ or $V_{S}$ at an incidence angle $\theta_{P}$ or $\theta_{S}$. Such excitation may be regarded as being broadly representative of that from either a surface 


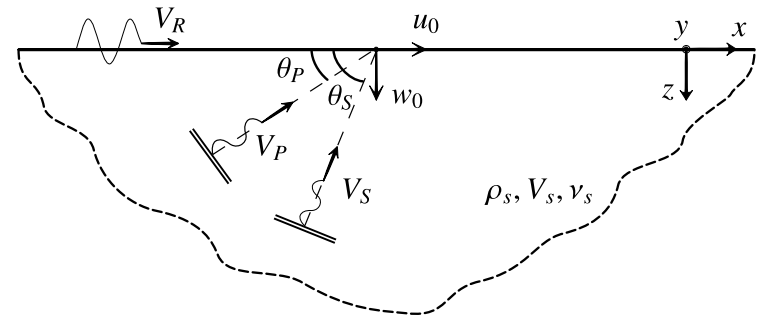

(a)

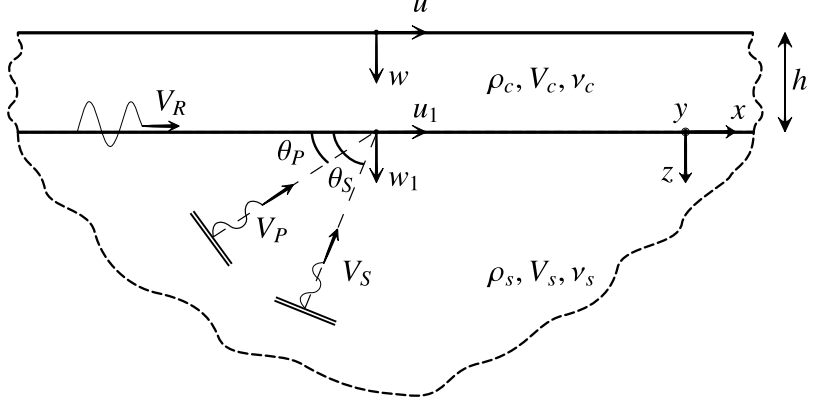

(b)

Figure 1: (a) The free-field displacement amplitude $\mathbf{u}_{0}$ on the surface of an elastic half-space subjected to surface and body planewave excitation. A Rayleigh wave travelling in the $\mathrm{x}$-direction with speed $V_{R}$, and a $\mathrm{P}$ - and $\mathrm{SV}$-wave travelling with speeds $V_{P}$ and $V_{S}$ at incidence angles $\theta_{P}$ and $\theta_{S}$ are considered; (b) the corresponding response following the construction of a slab foundation, expressed in terms of the interface displacement $\mathbf{u}_{\mathbf{1}}$ and the displacement $\mathbf{u}$ on the free-surface of the slab.

or underground railway.

The wave excitation is assumed to be plane and therefore invariant in the y-direction. The problem can then be examined in the $x-z$ plane with reference to the vertical and horizontal displacement amplitudes. The free-field displacement $\mathbf{u}_{\mathbf{0}}$ of the half-space, the interface displacement $\mathbf{u}_{\mathbf{1}}$ and the free-surface displacement $\mathbf{u}$ of the slab can be expressed in vector form as:

$$
\mathbf{u}_{\mathbf{0}}=\mathbf{A}_{\mathbf{0}} \exp \left\{i\left(\omega t-k_{x} x\right)\right\} ; \quad \mathbf{u}_{\mathbf{1}}=\mathbf{A}_{\mathbf{1}} \exp \left\{i\left(\omega t-k_{x} x\right)\right\} ; \quad \mathbf{u}=\mathbf{A} \exp \left\{i\left(\omega t-k_{x} x\right)\right\}
$$

where $\omega$ and $k_{x}$ are the angular frequency and horizontal wavenumber of the vibration field. The relative amplitudes of the vectors $\mathbf{A}_{\mathbf{0}}=\left[u_{0}, w_{0}\right]^{\mathrm{T}}, \mathbf{A}_{\mathbf{1}}=\left[u_{1}, w_{1}\right]^{\mathrm{T}}$ and $\mathbf{A}=[u, w]^{\mathrm{T}}$, which are independent of the $x$ coordinate due to the infinite extent of the slab, describe the influence of the slab foundation. Depending on the type of wave excitation considered, the horizontal wavenumber $k_{x}$ can be written as:

$$
k_{x}=k_{R} ; \quad k_{x}=k_{P} \cos \theta_{P} ; \quad k_{x}=k_{S} \cos \theta_{S}
$$

where $k_{R}, k_{P}$ and $k_{S}$ are the wavenumbers of the Rayleigh, P- and SV-waves respectively.

The influence of the slab foundation on the free-field displacement $\mathbf{u}_{\mathbf{0}}$ relates to a general result in the SSI literature [7]. So-called weak coupling is assumed, in which only the coupling between the soil and the slab is accounted for; any coupling with the original source of the vibration is assumed to be negligible. By ensuring equilibrium and compatibility at the soil-foundation interface, the displacement $\mathbf{u}_{\mathbf{1}}$ at the interface 
can be expressed as:

$$
\mathbf{u}_{\mathbf{1}}=\left[\mathbf{I}+\mathbf{H}_{\mathbf{s}} \mathbf{H}_{\mathbf{f}}^{-1}\right]^{-1} \mathbf{u}_{\mathbf{0}}
$$

where $\mathbf{H}_{\mathbf{f}}$ and $\mathbf{H}_{\mathrm{s}}$ are the frequency-response function (FRF) matrices of the foundation and soil respectively, which can be found by inverting the respective dynamic stiffness matrices [18]. Equation 3 is also found in the ground-borne vibration literature in the context of soil-building interaction [5]. In the case of finite slabs, Equation 3 may be solved directly; in the case of infinite slabs, the solution may be obtained via a frequency-wavenumber formulation. Auersch used the latter approach for an infinitely-long slab subjected to Rayleigh waves, and assumed a relaxed boundary condition between the soil and the slab in which only the vertical displacements at the interface are coupled [12]. In this case, Equation 3 reduces to the following scalar equation for the vertical displacement ratio at the interface:

$$
\frac{w_{1}}{w_{0}}=\frac{1}{\left(1+\frac{H_{s z}}{H_{f z}}\right)}
$$

where $H_{f z}$ and $H_{s z}$ are the vertical driving-point FRFs of the slab and the soil respectively, formally in the frequency-wavenumber domain. However, since the wavenumber is a function of frequency and the Rayleigh wave speed, this ratio of the displacement amplitudes is a function of frequency alone, and independent of position due to the infinite length of the plate.

Focussing on the vertical displacements is common practice when dealing with ground-borne vibration in buildings, often based on the assumption that the flexibility of a building in the horizontal direction provides sufficient decoupling from any horizontal vibration. However, there is theoretical evidence that suggests otherwise, with all coupling degrees-of-freedom (vertical, horizontal and rotational) between a building structure and its foundation being potentially significant [19]. Furthermore, given the small strains associated with ground-borne vibration, the influence of friction at the soil-slab interface may lead to a fully-coupled boundary condition that includes coupling of the horizontal displacements. In this case, the full matrix form of Equation 3 must be solved, although this does reduce to a scalar equation, similar to Equation 4, for the horizontal $u_{1} / u_{0}$ and vertical $w_{1} / w_{0}$ displacement ratios in the case of normally-incident SV- and P-waves respectively.

The fully-coupled condition calls into question the assumption of plate-like behaviour for the slab. By modelling the slab as a thin plate, the through-thickness deformation is assumed to be negligible, such that $w=w_{1}$, and there is no consideration given to the in-plane horizontal displacement. The aim of the current study is therefore to investigate how the SSI associated with a slab foundation is influenced by the slab-soil 
boundary condition and the plate-like assumption, and how this varies for the different incident wave fields.

Before presenting the results, it is helpful to review the dimensionality of the problem. By dimensional analysis, the displacement ratios $w / w_{0}, w_{1} / w_{0}$, etc. can each be expressed as a function of the following form:

$$
\frac{w}{w_{0}}=\Psi\left(\frac{V_{s}}{V_{c}}, \frac{\rho_{s}}{\rho_{c}}, v_{s}, v_{c}, \theta, \frac{\omega h}{V_{s}}\right)
$$

Assuming typical values for the Poisson's ratios $v_{s}$ and $v_{c}$ (Table A.1), the dimensionless groups $V_{s} / V_{c}$ and $\rho_{s} / \rho_{c}$ enable the influence of the stiffness and density of the slab relative to the soil to be investigated for a given wave incidence angle $\theta$ and non-dimensional frequency $a_{0}=\omega h / V_{s}$. The latter enables the thickness of the slab to be described relative to the shear wavelength in the soil. Given typical ranges for the shear wave speed in the soil $V_{s}=150-300 \mathrm{~m} / \mathrm{s}$, the slab thickness $h=0.5-1.5 \mathrm{~m}$ and the frequency range of ground-borne vibration $f=25-250 \mathrm{~Hz}$, the corresponding range of interest for the dimensionless frequency $a_{0}$ lies between approximately 0.2 and 15 .

\section{A slab foundation subjected to Rayleigh wave excitation}

This section explores the SSI associated with a slab foundation subjected to Rayleigh wave excitation. Before considering the slab as an elastic layer, the case of an infinitely long, strip foundation is first reviewed, following the approach of Auersch [12]. Having reproduced Auersch's results, the slab is then modelled as the elastic layer illustrated in Figure 1, enabling the influence of the slab-soil boundary condition and the slab's finite thickness to be investigated.

\subsection{The slab as an infinitely long, strip foundation}

The infinitely long, strip foundation is modelled as an elastic plate of width $b$ and thickness $h$. The vertical dynamic stiffness of the plate in the frequency-wavenumber domain is given by [20]:

$$
K_{f z}\left(\omega, k_{x}\right)=B b k_{x}^{4}-\rho_{c} b h \omega^{2}
$$

where $B=E_{c} h^{3} / 12\left(1-v_{c}^{2}\right)$ is the bending stiffness. The vertical driving-point FRF is obtained as $H_{f z}=$ $1 / K_{f z}$.

The vertical driving-point FRF of the elastic half-space $H_{s z}$ refers to a 2.5-D problem because of the finite width $b$ of the plate in the y-direction. Auersch solved this by assuming plane-strain conditions and using a numerical integration approach in the wavenumber $k_{y}$, regarding the plate as infinitely flexible along 
the $y$-axis by assuming a constant stress distribution across the width of the plate. According to the relaxedboundary condition, the vertical displacement ratio $w_{1} / w_{0}$ is then obtained from Equation 4

Figure 2 plots the ratio $w_{1} / w_{0}$ as a function of frequency for the parameter values of the benchmark problem considered by Auersch (see Table A.1 in Appendix A. Several values of the foundation width $b$ are considered. Irrespective of the width, at a particular frequency known as the "coincidence frequency" $\left(f_{c o}\right)$, the bending stiffness and inertia of the plate are such that a unit value of $w_{1} / w_{0}$ is obtained. The coinci-

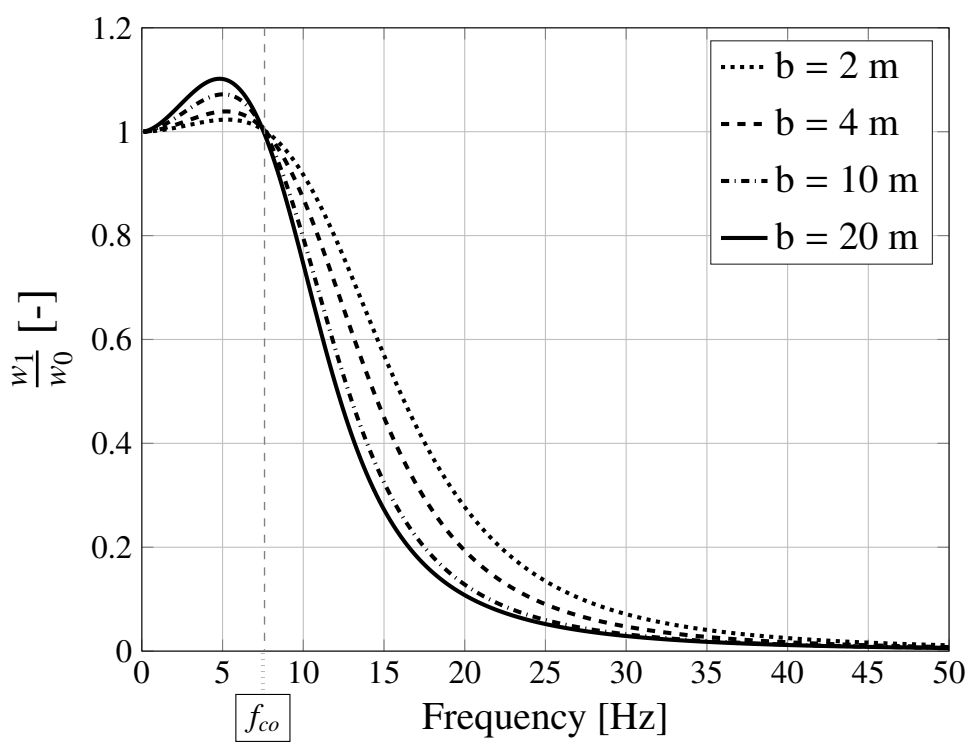

Figure 2: The influence of the width of a strip foundation $b$ on the soil-foundation interaction under Rayleigh wave excitation, as described by the ratio of the vertical displacement amplitudes at the soil-foundation interface, before $\left(w_{0}\right)$ and after $\left(w_{1}\right)$ the construction of the foundation. Benchmark parameter values (Table A.1.

dence point represents the scenario in which the free-flexural wavelength $\lambda_{f}$ of the plate coincides with the horizontal wavelength $\lambda_{x}$ of the input wave-field. The corresponding frequency can be found as [20]:

$$
f_{c o}=\frac{V_{R}^{2}}{2 \pi} \sqrt{\frac{\rho_{c} h}{B}}=\frac{V_{R}^{2}}{2 \pi} \sqrt{\frac{\rho_{c} 12\left(1-v_{c}^{2}\right)}{E_{c} h^{2}}}
$$

The 
$w_{1} / w_{0}$ (for frequencies $f>f_{c o}$ ). A similar effect may be obtained by decreasing the mass density $\rho_{c}$ of the plate. A comprehensive discussion of the influence of the bending stiffness, mass and width of the strip foundation, and the shear wave speed $V_{s}$ of the soil, can be found in Auersch [12], also with reference to a finite plate. The results of Figure 2 also illustrate the significance of increasing the strip width $b$, with the limiting case being that of a slab foundation of infinite extent along the $\mathrm{x}$ - and $\mathrm{y}$-axis $(b \rightarrow \infty)$.

\subsection{The slab as an elastic layer}

We now assume the slab foundation to be of infinite extent in both the $x$ and $y$ direction, modelling it as an elastic layer of finite thickness overlying the elastic half-space. The dynamic stiffness matrices $\mathbf{K}_{\mathbf{s}}$ and $\mathbf{K}_{\mathbf{f}}$ of both the half-space and the layer are calculated by means of the dynamic stiffness method (DSM) [18], making use of the ElastoDynamics Toolbox (EDT) [13, 14] in MATLAB [15]. The FRF matrices appearing in Equation $3, \mathbf{H}_{\mathbf{s}}$ and $\mathbf{H}_{\mathbf{f}}$, are obtained by inverting the respective dynamic stiffness matrices.

In the case of the relaxed boundary (RB) condition, the vertical displacement ratio $w_{1} / w_{0}$ can be retrieved from Equation 4, with the vertical driving-point FRFs, $H_{s z}$ and $H_{f z}$, being extracted from the FRF matrices. Figure 3 a plots the ratio $w_{1} / w_{0}$ calculated by both Auersch's strip model and the equivalent
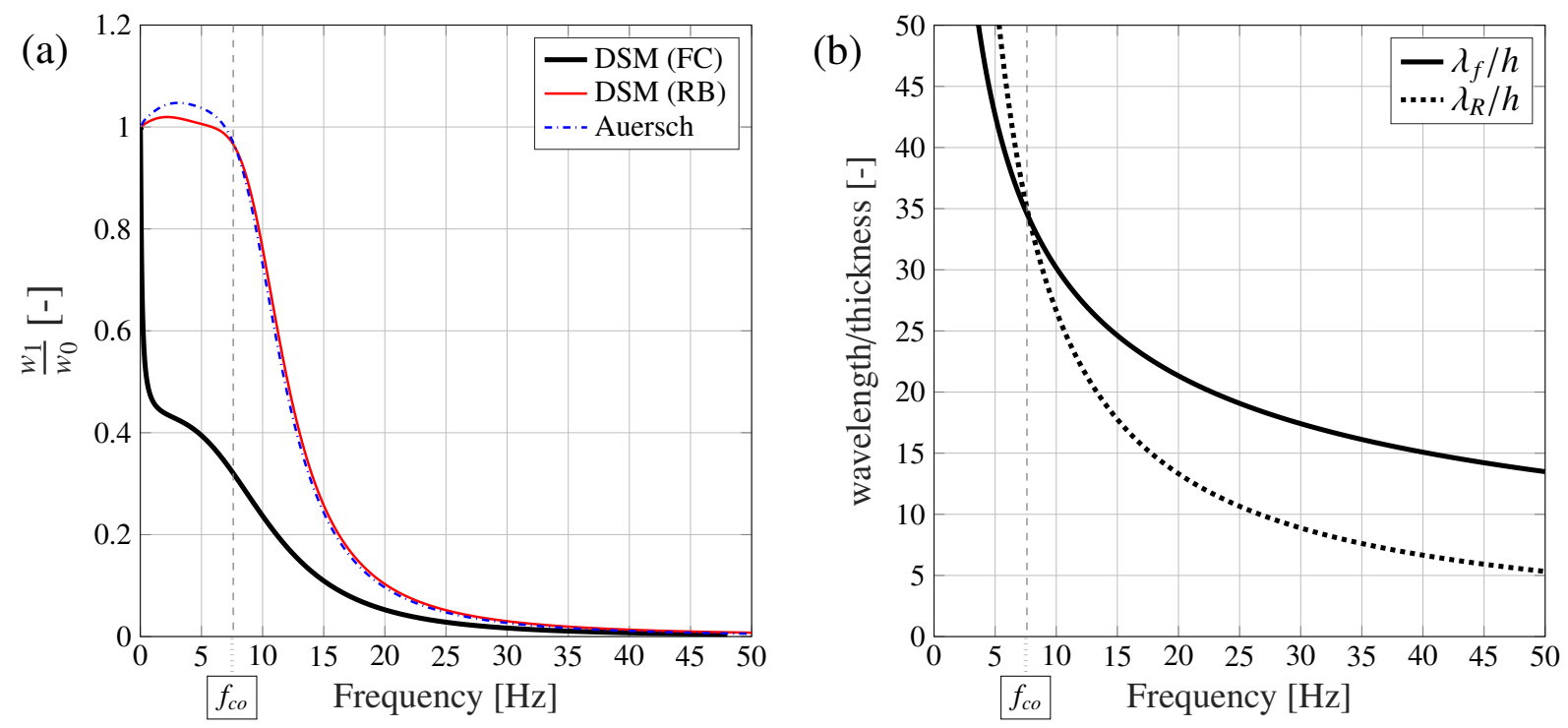

Figure 3: (a) The vertical displacement ratio at the soil-foundation interface of an infinitely long (Auersch) and infinitely large (DSM) slab foundation subjected to an incident Rayleigh wave, considering the fully-coupled (FC) and relaxed (RB) boundary conditions. (b) Comparison of the Rayleigh wave-field wavelength $\lambda_{R}$ and the free-flexural wavelength of the strip foundation $\lambda_{f}$. Benchmark parameter values (Table A.1. 
DSM (RB) model, for the same benchmark parameter values (Table A.1). It is clear that the two different methods agree well, with the strip model achieving convergent results with the DSM (RB) model for a slab width of $b=1000 \mathrm{~m}$. Figure $3 \mathrm{~b}$ plots the wavelength-frequency curves associated with the Rayleigh wavefield and the free-flexural response of the plate model. The coincidence point again indicates the transition region from amplification to attenuation.

In the fully-coupled condition (FC), the displacement ratios $w_{1} / w_{0}$ and $u_{1} / u_{0}$ are obtained by calculating the interface displacement $\mathbf{u}_{\mathbf{1}}$ using Equation 3, with reference to both the horizontal and vertical components of the Rayleigh wave [21]. The ratio $w_{1} / w_{0}$ obtained with this DSM (FC) model is also plotted in Figure 3 a. In this condition, the SSI leads to significant attenuation for all frequencies, without any amplification below the coincidence frequency. The fully-coupled condition highlights the importance of considering both the horizontal and vertical components of $\mathbf{u}_{\mathbf{0}}$, and the cross-stiffness terms at the soilfoundation interface.

Figure 4 4 plots the horizontal displacement ratio $u_{1} / u_{0}$, calculated by the DSM model for both the relaxed (RB) and fully-coupled (FC) conditions. The common feature is that both conditions result in a significant attenuation of the horizontal displacement. Again, the assumption of plane-wave excitation sim-
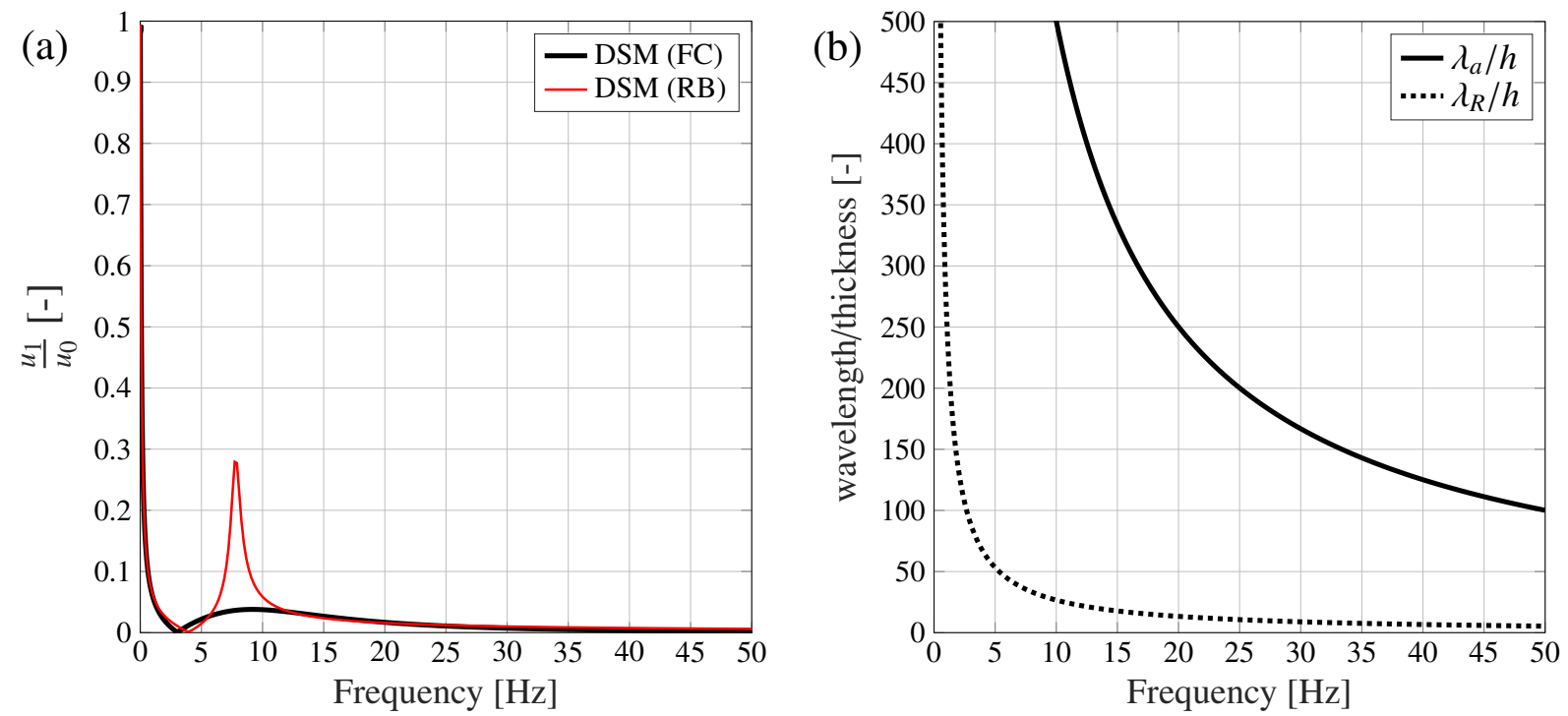

Figure 4: (a) The horizontal displacement ratio at the soil-foundation interface of an infinitely large slab foundation subjected to an incident Rayleigh wave, considering the fully-coupled (FC) and relaxed (RB) boundary conditions. (b) Comparison of the Rayleigh wave-field wavelength $\lambda_{R}$ and the free-axial wavelength of the slab $\lambda_{a}$. Benchmark parameter values (Table A.1). 


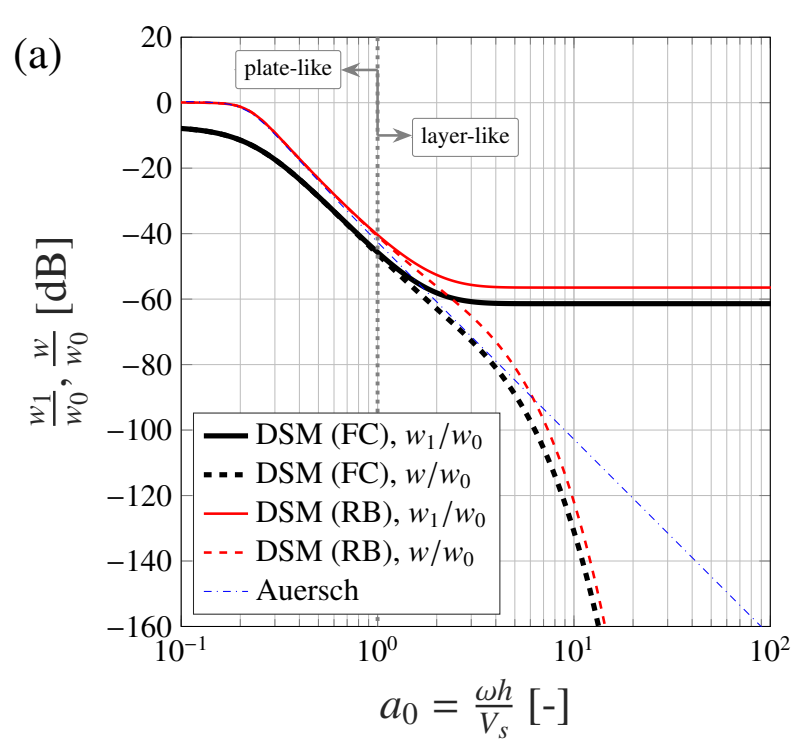

plifies the problem and enables a physical interpretation of the SSI. From the perspective of the (horizontal) slab, the incident wave-field is characterised by a horizontal wavelength $\lambda_{x}=\lambda_{R}$. This is true for the horizontal displacement $u_{1}$ as for the vertical displacement $w_{1}$ seen earlier. The horizontal displacement of the slab is influenced by its free-axial response along the $x$ direction, the wavelength of which is given by:

$$
\lambda_{a}=\frac{1}{f} \sqrt{\frac{E_{c}}{\rho_{c}\left(1-v_{c}^{2}\right)}}
$$

Figure $4 \mathrm{~b}$ plots this wavelength-frequency relationship together with the curve for the Rayleigh wave-field. It is clear that $\lambda_{R}$ is always shorter than $\lambda_{a}$, with no coincidence possible. It follows that the axial (in-plane) behaviour of the slab always restrains the horizontal displacement at the soil-foundation interface, resulting in attenuation for all frequencies for both the fully-coupled and relaxed boundary conditions.

A better understanding of the results from the different models may be obtained by expressing the displacement ratios in decibels $(\mathrm{dB})$ and referring to the non-dimensional frequency $a_{0}$ on a logarithmic scale. Figure $5 \mathrm{a}$ re-plots in this form the results of Figure 3 a for the vertical displacement ratio at the soilfoundation interface. Also included in Figure $5 \mathrm{a}$ are the results for the displacement ratio $w / w_{0}$ calculated

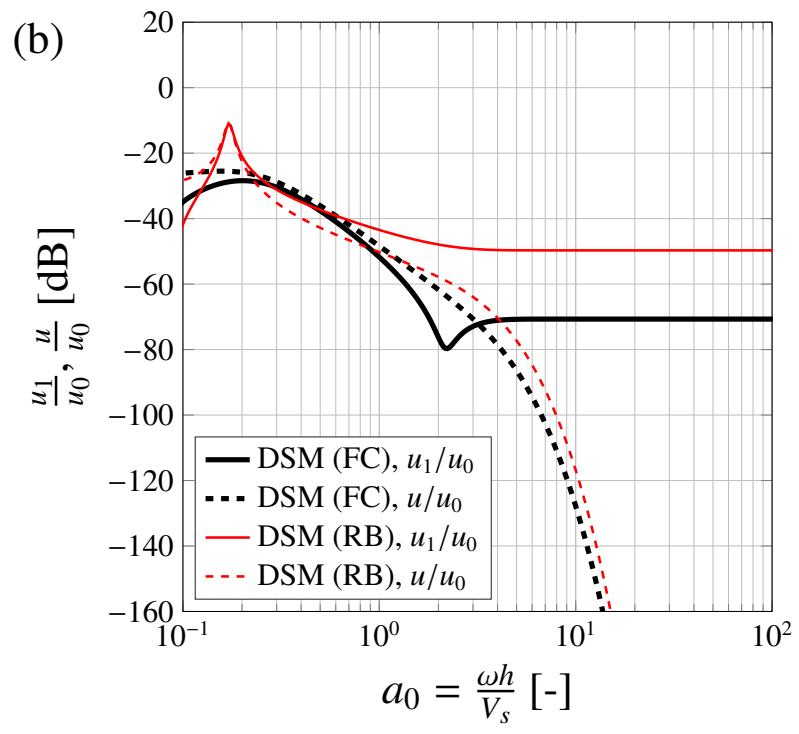

Figure 5: (a) The vertical displacement ratio at the free-surface $\left(w / w_{0}\right)$ and soil-foundation interface $\left(w_{1} / w_{0}\right)$ of an infinitely large slab foundation subjected to an incident Rayleigh wave, considering the fully-coupled (FC) and relaxed (RB) boundary conditions. Comparison is made with Auersch's strip model. (b) The corresponding horizontal displacement ratios. Benchmark parameter values (Table A.1) 
at the free-surface - the location of most interest, since this will support any future building. The platelike behaviour of the slab at relatively low frequencies is clearly captured by the Auersch strip model, which shows a good agreement with the DSM (RB) model up to $a_{0} \approx 1$. The plate-like assumption is therefore sufficient to capture the SSI, as observed at the soil-slab interface (ratio $w_{1} / w_{0}$ ) with the relaxed boundary condition, but only up to a restricted value of $h / \lambda_{x}$. For shorter wavelengths, the through-thickness effects become more important, with the layer-like behaviour of the slab attenuating the wave field at the interface $w_{1}$ down to a plateau at high frequencies. This is a common feature for both the relaxed and fully-coupled boundary conditions, although the former leads to lower attenuation because it neglects the retrograde elliptical motion of particles at the soil-foundation interface, characteristic of Rayleigh waves, accounting for only the vertical component. At the free surface, the high-frequency plateau is not evident. Instead, the level of attenuation is observed to increase with frequency. In the case of the relaxed boundary condition, the vertical displacement $w$ is obtained simply as:

$$
\frac{w}{w_{1}}=\frac{\widetilde{H}_{f z}}{H_{f z}}
$$

where $\widetilde{H}_{f z}$ is the FRF for the vertical displacement at the free-surface due to a vertical traction at the interface, which can be retrieved from the FRF matrix of the layer $\mathbf{H}_{\mathbf{f}}$. Again, it is clear from Equation 9 that the DSM (RB) model neglects the coupling between the horizontal and the vertical motion. The ratio $w / w_{1}$ approaches unity at low frequencies, when the through-thickness effects are negligible, but introduces increasing attenuation at higher frequencies.

Figure $5 \mathrm{~b}$ plots the corresponding results for the horizontal displacements, this time re-plotting the results of Figure $4 a$ for the displacement ratio at the interface $u_{1} / u_{0}$, together with the free-surface ratio $u / u_{0}$. A similar trend to the vertical displacements is observed but with the exception of the clearly absent amplification region at low frequencies for the DSM (RB) model, due to the lack of any coincidence phenomenon.

For both the vertical and the horizontal displacements, the results of the DSM (RB) and DSM (FC) models have common features that indicate the importance of both the finite thickness of the slab, which becomes increasingly significant with frequency, and the assumed soil-foundation boundary condition. The influence of the latter results in at least $10 \mathrm{~dB}$ difference in the final free-surface displacements of the slab between the two conditions, without influencing the general trend in the results. 


\section{A slab foundation subjected to incident P-and SV-waves}

So far, the SSI associated with a slab foundation has only been investigated in the context of Rayleigh waves. The case of incident P- or SV-waves can be similarly investigated by using the same DSM model for the elastic layer but now with the free-field displacement components associated with these wave types [21]. Considering an incident $\mathrm{P}$ - or SV-wave at an incidence angle $\theta_{P}$ or $\theta_{S}$ (see Figure 1), the displacement field $\mathbf{u}$ of the coupled soil-foundation system is calculated by the superposition of several elastic states [22]. Only the fully-coupled slab is considered here, in the belief that this better represents the actual soil-foundation boundary condition at the low strain levels present in practice.

\subsection{Influence on the vertical displacements $\left(w_{1} / w_{0}\right.$ and $\left.w / w_{0}\right)$}

Figure 6 plots the interface displacement ratio $w_{1} / w_{0}$, together with the wavelength-frequency curves for incident P-waves (Figure 6a and 6c) and SV-waves (Figure 6b and 6d), for the benchmark parameter values (Table A.1) and an arbitrary incidence angle $\theta_{P}=\theta_{S}=3 \pi / 8$. The results in this particular case can be qualitatively divided into the following frequency regions:

1. an initial low-frequency region of either moderate attenuation (for P-waves) or amplification (for $\mathrm{SV}$-waves), up to a frequency $f_{1}<f_{c o}$;

2. a second region, with increased values of $w_{1} / w_{0}$, which are always greater than unity for SV-waves but not necessarily for P-waves. A maximum value is obtained at a frequency $f_{2}>f_{c o}$;

3. a third region of sharply increasing attenuation, up to a frequency $f_{3}$, beyond which $w_{1} / w_{0}$ tends to a limiting value.

The wavelength-frequency curves (Figure 6c and 6d) are again helpful for interpreting the SSI. The coincidence frequency $f_{c o}$ lies between $f_{1}$ and $f_{2}$, and does not correspond to a distinct feature in the $w_{1} / w_{0}$ curve. This is because the free-flexural wavelength $\lambda_{f}$ of the slab is calculated based on the thin-plate assumption; in practice, shear deformation is likely to contribute to higher values of $\lambda_{f}$, resulting in lower values of $f_{c o}$.

As seen before, a better understanding of the results is obtained by using logarithmic scales. Figure 7 shows such a representation for the results in Figure 6 (a) and (b). The trend in the ratio $w_{1} / w_{0}$ described before can be observed more clearly with reference to the local minimum and maximum at $a_{0}^{(1)}$ and $a_{0}^{(2)}$, and the plateau beyond $a_{0}^{(3)}$. Additionally, the free-surface displacement ratio $w / w_{0}$ is plotted. Analogous to what is observed for Rayleigh wave excitation, additional attenuation is obtained for the free-surface 
(a)

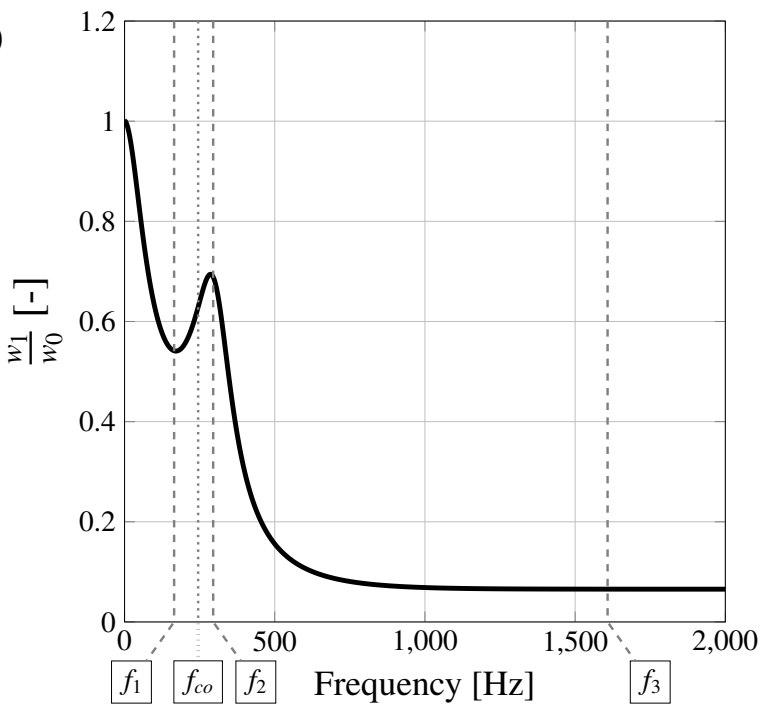

(c)

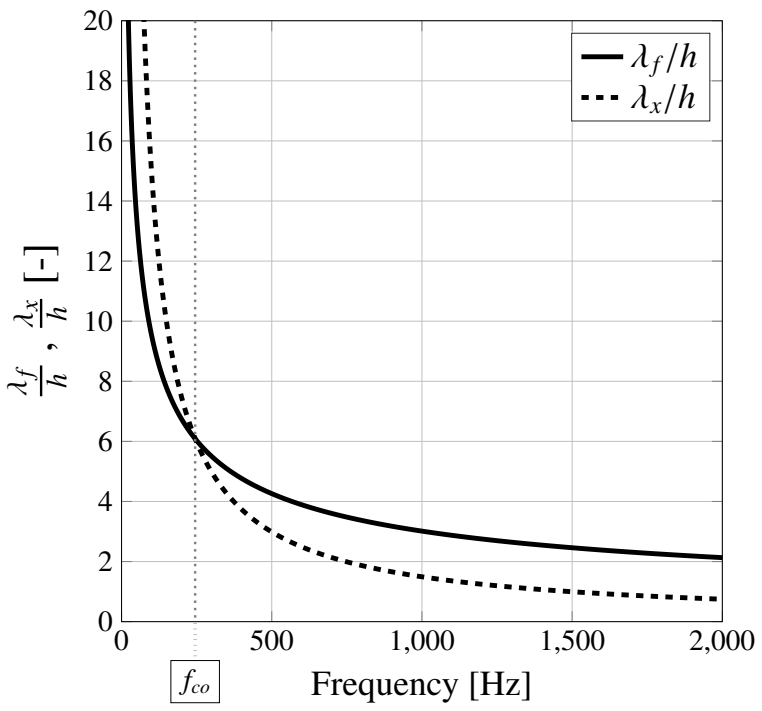

(b)

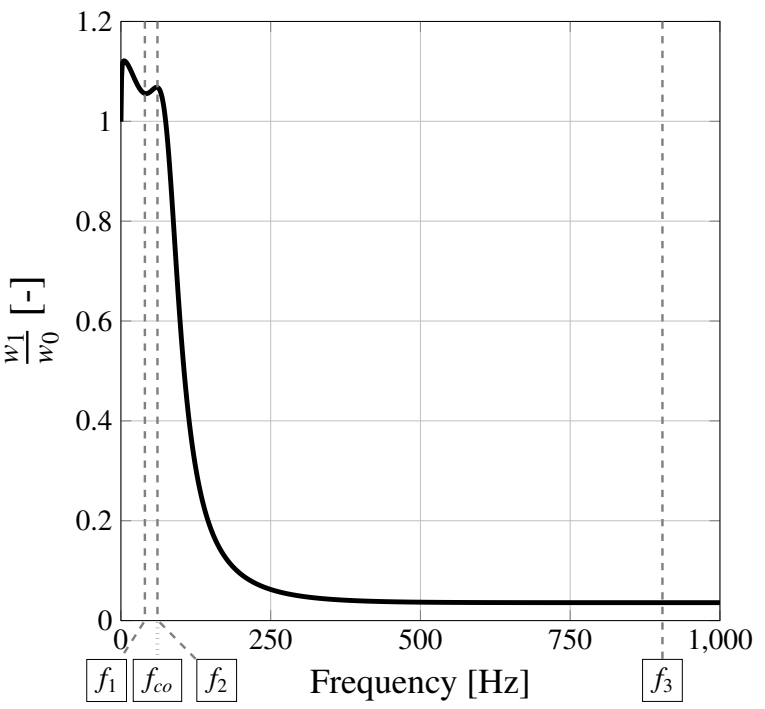

(d)

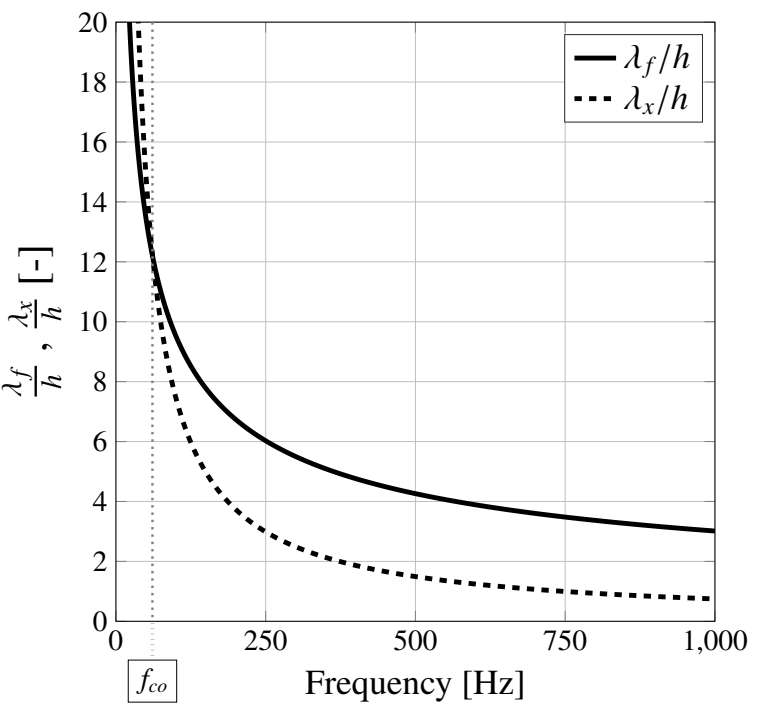

Figure 6: The vertical displacement ratio $w_{1} / w_{0}$ at soil-foundation interface of an infinitely large slab foundation subjected to (a) an incident P-wave at an angle $\theta_{P}=3 \pi / 8$ and (b) an incident SV-wave at an angle $\theta_{S}=3 \pi / 8$. The wavelength-frequency curves are plotted below for the two cases ((c) P- and (d) SV-waves) with reference to the coincidence frequency $f_{c o}$ based on the thin-plate assumption. Benchmark parameter values (Table A.1).

displacement $w$, compared to the interface displacement $w_{1}$, as a result of through-thickness effects at relatively short wavelengths. The results obtained using Auersch's thin-plate assumption are also included to highlight the influence of the relaxed boundary condition and the thin-plate assumptions.

Under Rayleigh wave excitation, investigated in Section 3, the attenuation provided by the fully-coupled 

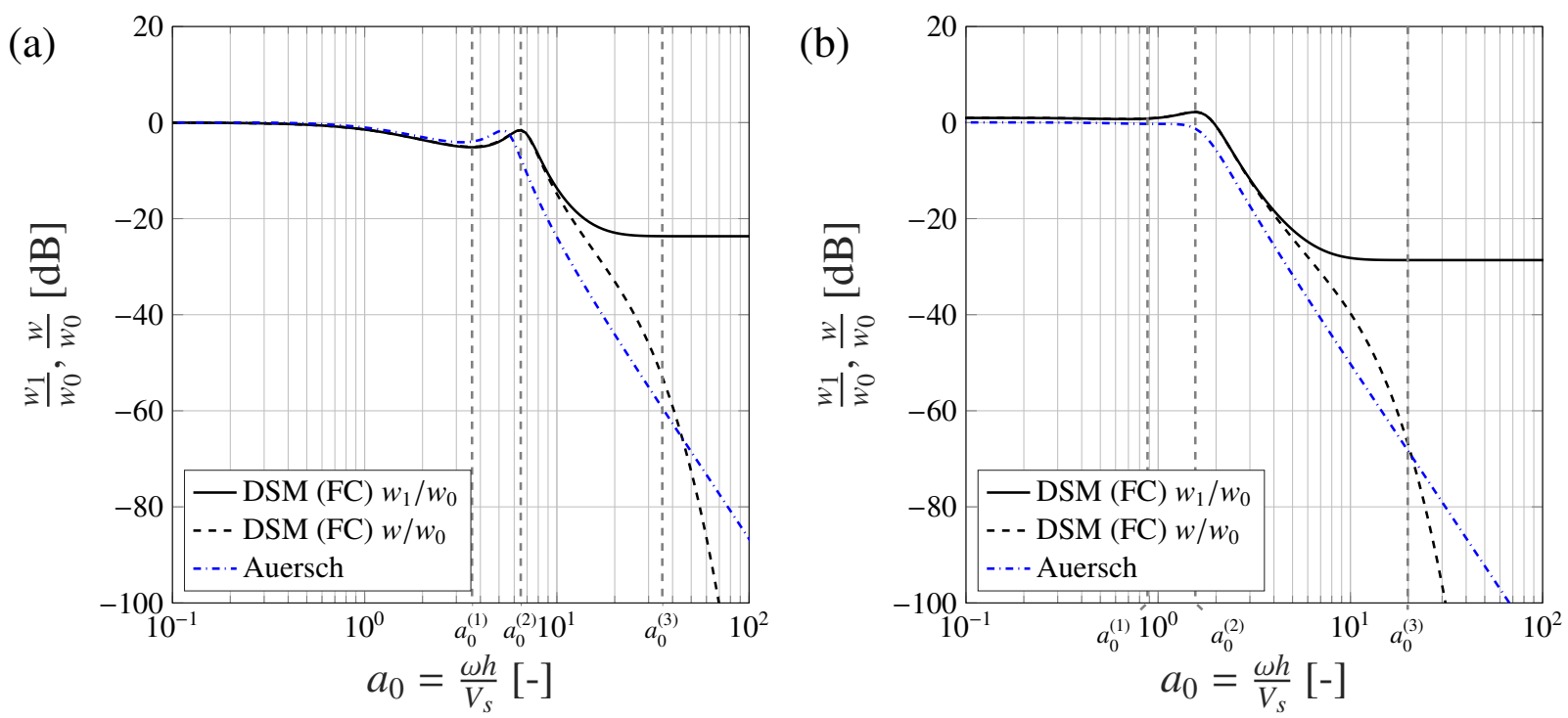

Figure 7: The vertical displacement ratio at the free-surface $\left(w / w_{0}\right)$ and soil-foundation interface $\left(w_{1} / w_{0}\right)$ of an infinitely large slab foundation subjected to (a) an incident P-wave at an angle $\theta_{P}=3 \pi / 8$ and (b) an incident SV-wave at an angle $\theta_{S}=3 \pi / 8$. Results obtained using Auersch's thin-plate assumption are included for comparison. Benchmark parameter values (Table A.1.

slab is considerable for all frequencies, and significantly greater than that provided to P- and SV-waves. The horizontal wavelength $\lambda_{x}$ associated with an incident P- or SV-wave is always longer than that of a Rayleigh wave of the same frequency (see Figure 6 and 6d). Consequently, the coincidence frequencies $f_{c o}$ associated with P- and SV-waves are always higher than that of the Rayleigh wave, and the region where strong attenuation is to be expected from the slab shifts to relatively high frequencies. The attenuation provided by the slab to Rayleigh waves therefore represents a limiting case.

Another limiting case is that of normally incident P-waves $\left(\theta_{P}=\pi / 2\right)$. In this case $k_{x}=0$ and the restraining effect of the slab foundation is maximum at the free-axial natural frequencies $f_{n}$ of the elastic layer in the vertical direction:

$$
f_{n}=\frac{V_{P c}}{4 h}(2 n-1)
$$

where $V_{P c}$ is the compressional wave speed in the slab. At these frequencies, the slab behaves like a dynamic vibration absorber. However, given the application, and the relatively high P-wave speed $V_{P c}, f_{n}$ lies well beyond the frequency range of interest $\left(f_{n=1} \approx 1270 \mathrm{~Hz}\right.$ for the parameters in Table A.1), leading to only moderate attenuation in the frequency range of interest. 


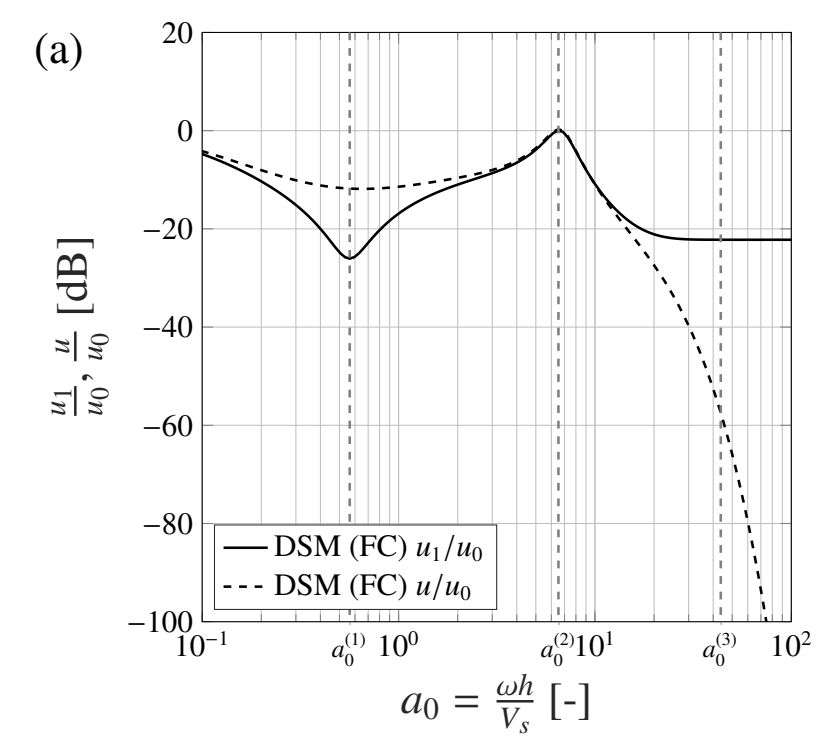

\subsection{Influence on the horizontal displacements $\left(u_{1} / u_{0}\right.$ and $\left.u / u_{0}\right)$}

A similar trend is observed in the horizontal displacements. This is illustrated in Figure 8 for incident $\mathrm{P}$ - and SV-waves at an arbitrary incidence angle $\theta=3 \pi / 8$. Although, by physical interpretation, no coincidence

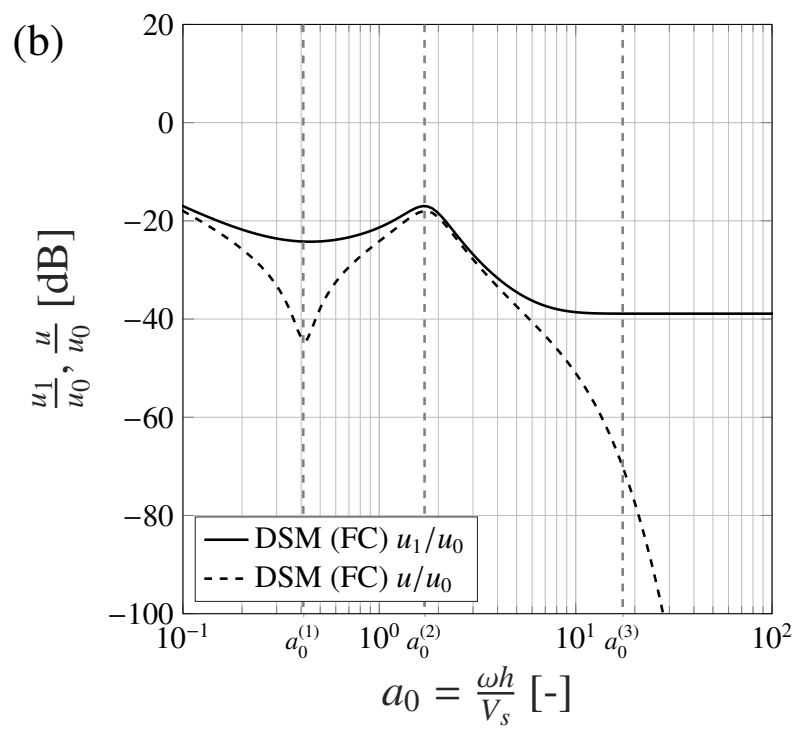

Figure 8: The horizontal displacement ratio at the free-surface $\left(u / u_{0}\right)$ and soil-foundation interface $\left(u_{1} / u_{0}\right)$ of an infinitely large slab foundation subjected to (a) an incident P-wave at an angle $\theta_{P}=3 \pi / 8$ and (b) an incident SV-wave at an angle $\theta_{S}=3 \pi / 8$. Benchmark parameter values (Table A.1.

phenomenon is expected in this case (see Section 3.1 , local minima $\left(a_{0}^{(1)}\right)$ and maxima $\left(a_{0}^{(2)}\right)$ are observed for the ratios $u_{1} / u_{0}$ and $u / u_{0}$. Moreover, the through-thickness effects at relatively short wavelengths result, once more, in additional attenuation of the free-surface displacement $u$. Following a similar argument to that used for the vertical displacements, it is clear that the Rayleigh wave and the normally incident SV-wave represent limiting cases for the horizontal displacements $u_{1}$ and $u_{0}$.

\subsection{The case of normally incident $P$ - and/or $S V$-waves}

The case of normally incident waves is worth exploring further, since these may be representative of the wave field from a deep source, such as an underground railway. For normal incidence, the wave propagation problem is independent of $x$ and effectively one-dimensional. The free-surface and interface displacement amplitudes $\mathbf{u}$ and $\mathbf{u}_{\mathbf{1}}$ can be found, in terms of the incident wave amplitude, by considering the superposition of multiple reflections and transmissions in the half-space-layer system, as illustrated schematically in 
Figure 9 It is clear that a normally incident P- or SV-wave will induce only vertical or horizontal displacements respectively. With a similar discussion also being valid for the case of a normally incident SV-wave, the notation adopted in the following refers only to a normally incident P-wave (see Appendix B.).

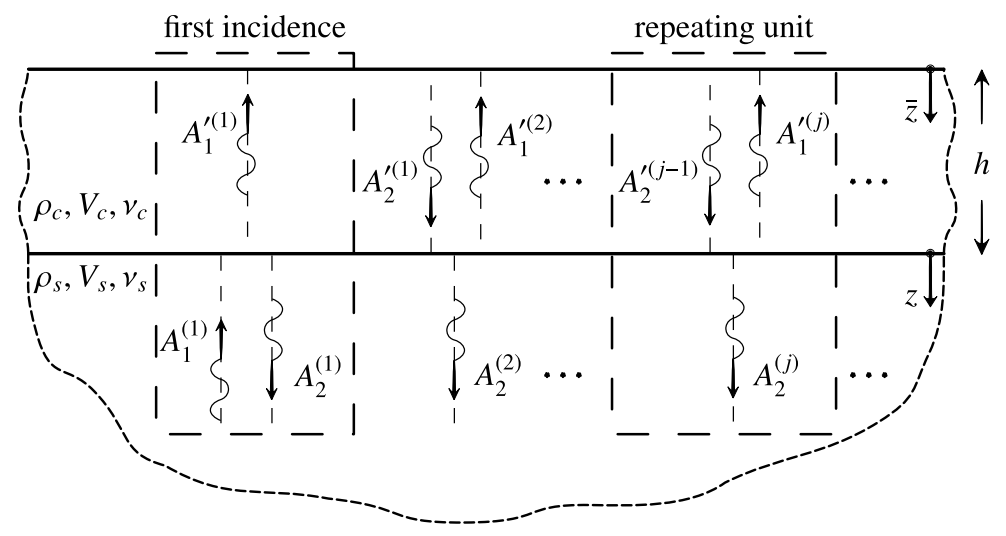

Figure 9: Schematic representation of the multiple reflected-transmitted wave amplitudes in an elastic layer overlying an elastic half-space, due to a normally incident P-wave. An infinite repetition of a reflection-transmission unit follows the reflection and transmission of the incident amplitude $A_{1}^{(1)}$.

As shown in Figure 9, the multiple reflections/transmissions trace back to the incidence of the amplitude $A_{1}^{(1)}$ at the interface, and to the repeating reflection/transmission of the amplitude $A_{2}^{\prime(j-1)}$ that results from the reflection of $A_{1}^{\prime(j-1)}$ at the free-surface. In general, the vertical displacement in the layer can be written as:

$$
w(\bar{z})=i k_{P}^{\prime}\left(\sum_{j=1}^{\infty} A_{1}^{\prime(j)} \exp \left(i k_{P}^{\prime} \bar{z}\right)-\sum_{j=1}^{\infty} A_{2}^{(j)} \exp \left(-i k_{P}^{\prime} \bar{z}\right)\right)
$$

By ensuring equilibrium and compatibility at the interface for the two cases, one can find:

$$
\frac{A_{2}^{(1)}}{A_{1}^{(1)}}=\frac{\beta_{P}-1}{\beta_{P}+1}
$$

$$
\frac{A_{1}^{\prime(j)}}{A_{2}^{\prime(j-1)}}=\frac{1-\beta_{P}}{\beta_{P}+1} \exp \left(-i 2 k_{P}^{\prime} h\right)=\zeta
$$

$$
\frac{A_{1}^{\prime(1)}}{A_{1}^{(1)}}=\frac{k_{P}}{k_{P}^{\prime}} \frac{2}{\beta_{P}+1} \exp \left(-i k_{P}^{\prime} h\right)
$$

$$
\frac{A_{2}^{(j)}}{A_{2}^{\prime(j-1)}}=\frac{k_{P}^{\prime}}{k_{P}} \frac{2 \beta_{P}}{\beta_{P}+1} \exp \left(-i k_{P}^{\prime} h\right)
$$

where $\beta_{P}=\left(\rho_{c} V_{P c}\right) /\left(\rho_{s} V_{P s}\right)$. The amplitude $A_{2}^{\prime(j)}=-A_{1}^{\prime(j)}$ because of the stress-free condition at $\bar{z}=0$. It is clear that all the amplitudes involved in the repeating unit in Figure 9 trace back to the amplitude $A_{1}^{\prime(1)}$ and 
that the latter refers to $A_{1}^{(1)}$. The vertical displacement at the free-surface can then be found as:

$$
w(\bar{z}=0)=i k_{P}^{\prime}\left(\sum_{j=1}^{\infty} A_{1}^{\prime(j)}-\sum_{j=1}^{\infty} A_{2}^{\prime(j)}\right)=2 i k_{P}^{\prime} A_{1}^{\prime(1)}\left(1-\zeta+\zeta^{2}+\ldots\right)=\frac{2 i k_{P}^{\prime} A_{1}^{\prime(1)}}{1+\zeta}
$$

Substituting the terms $\zeta$ and $A_{1}^{\prime(1)}$, and considering the free-field amplitude $w_{0}$ from Appendix B

$$
\frac{w}{w_{0}}=\frac{1}{\cos \left(k_{P}^{\prime} h\right)+i \beta_{P} \sin \left(k_{P}^{\prime} h\right)}
$$

With a similar argument for the interface displacement $w_{1}$, we can write:

$$
\frac{w_{1}}{w_{0}}=\frac{1}{1+i \beta_{P} \tan \left(k_{P}^{\prime} h\right)}
$$

From the latter, as anticipated from Equation 10, it is clear that the slab has a restraining effect on the interface displacement $w_{1}$ at the free-axial natural frequencies $f_{n}$ of the elastic layer. This effect disappears at frequencies $2 f_{n}$, when $w_{1}=w_{0}$ if damping is neglected. The latter are frequencies for which the halfwavelength of the P-waves, or a multiple, matches the slab foundation thickness. In this case, the ratios $w / w_{0}$ and $w_{1} / w_{0}$ will converge to the same value, that is, unity in the undamped case. In general, for

(a)

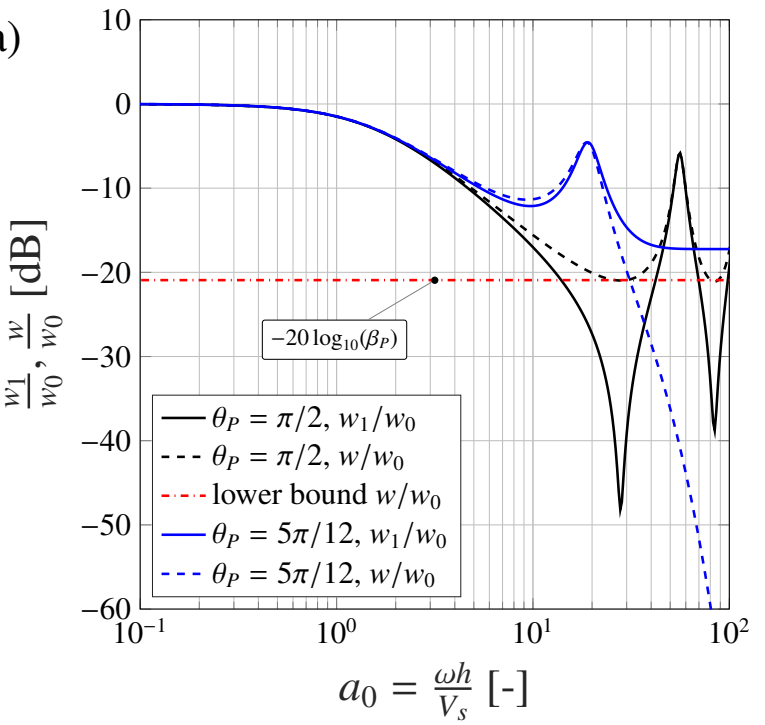

(b)

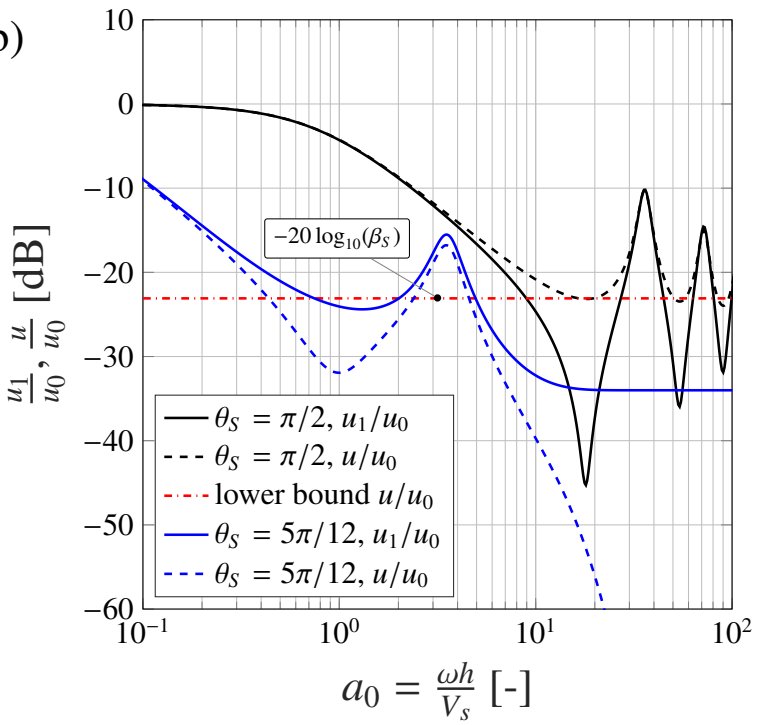

Figure 10: (a) The vertical displacement ratios at the free-surface $\left(w / w_{0}\right)$ and soil-foundation interface $\left(w_{1} / w_{0}\right)$ of an infinitely large slab foundation subjected to an incident P-wave at angles of $\pi / 2$ and $5 \pi / 12$. (b) The equivalent horizontal displacement ratios due to an incident SV-wave. Benchmark parameter values (Table A.1).

the case of normal incidence, the free-surface displacement $w$ is greater than, or at least equal to, the 
interface displacement $w_{1}$. The behaviour illustrated here depends only on the relative characteristics of the soil-slab system, that is, the impedance ratio $\beta$. This is generally greater than unity for both $\mathrm{P}$-waves, $\beta_{P}=\left(\rho_{c} V_{P c}\right) /\left(\rho_{s} V_{P s}\right)$, and SV-waves, $\beta_{S}=\left(\rho_{c} V_{S c}\right) /\left(\rho_{s} V_{s}\right)$, so that the slab has an overall attenuating effect on the displacement amplitudes. In contrast to the interface displacement, for the free-surface displacement $w$ such attenuation has a limiting value, again related to the impedance ratio $\beta$. This is shown in Figure 10a for normally incident P-waves and, in Figure 10 p, for normally incident SV-waves. Figure 10 also shows the results for an incidence angle just off normal, that is, $\theta=5 \pi / 12$.

It is clear that normal incidence represents a special case, when the slab behaves as a simple elastic layer, with no plate-like behaviour. For both P- and SV-waves, this ensures no amplification of the incident waves but limits the available attenuation. Once the incidence angle deviates from normal, even by a small amount, plate-like behaviour becomes significant, resulting in additional attenuation that increases with frequency.

\section{Design of slab foundations against ground-borne vibration}

This section considers the overall implications of this study for foundation design. In particular, it considers what guidance may be drawn regarding the design of a slab foundation to achieve a specific reduction in ground-borne vibration level. It is clear that the SSI associated with a slab foundation is complex, even for the simplified system considered here. Nevertheless, some useful guidance may be presented in the form of Figures 11, 12 and 13 . These present a series of summary design plots that illustrate the influence of the dimensionless groups $V_{s} / V_{s c}, \rho_{s} / \rho_{c}$ and $\theta$, over the typical ranges associated with ground-borne vibration, for the three incident wave types (P, SV and Rayleigh). Typical values are assumed for the Poisson's ratios and damping loss factors of the slab and soil (Table A.1). The results are given in terms of the displacement ratios at the free-surface of the slab, for the fully-coupled boundary condition at the soil-foundation interface.

\subsection{Surface Vibration Sources}

In the case of a surface source, it is reasonable to assume that the incident vibration field will be dominated by Rayleigh waves, at least for locations remote from the source. In this case, the plots in Figure 13 summarise concisely the behaviour of the slab. It is clear that the density ratio has a relatively weak influence, with the level of attenuation varying by no more than $6 \mathrm{~dB}$ over the typical range $\rho_{s} / \rho_{c}=0.6-1.2$. A relatively dense slab is therefore desirable but probably not worth pursuing actively, given the expense of 
specialist high-density concrete. In contrast, the wave speed ratio has a strong influence, with the attenuation varying by up to $25 \mathrm{~dB}$ over the typical range $V_{s} / V_{s c}=0.05-0.2$. This sensitivity to the wave speed ratio indicates the significance of the relative stiffness of the slab, and the importance of obtaining an estimate of soil stiffness before relying on a particular slab for vibration mitigation.

For a given site, the controlling parameter is the slab thickness, which the designer is free to select in order to provide acceptable attenuation at the lowest frequency of concern, in the knowledge that any higher frequencies are attenuated further. For example, the results indicate that, for the benchmark soil properties, typical of London Clay $\left(V_{s}=200 \mathrm{~m} / \mathrm{s}, \rho_{s}=2000 \mathrm{~kg} / \mathrm{m}^{3}\right)$, a concrete $\mathrm{slab}\left(V_{s c}=2284 \mathrm{~m} / \mathrm{s}, \rho_{c}=2500 \mathrm{~kg} / \mathrm{m}^{3}\right)$ of thickness $0.5 \mathrm{~m}$ would provide $24 \mathrm{~dB}$ of attenuation to vertical vibration at $25 \mathrm{~Hz}$, increasing to $81 \mathrm{~dB}$ at $250 \mathrm{~Hz}$. The corresponding attenuation of horizontal vibration is slightly greater, ranging from $33 \mathrm{~dB}$ to $79 \mathrm{~dB}$

\subsection{Buried Sources}

In the case of a buried source, when body waves are more significant, the situation is more complex. As with Rayleigh excitation, the attenuation provided by the slab is influenced only weakly by the density ratio (again, by no more than $6 \mathrm{~dB}$ ) but strongly by the wave speed ratio, this time by up to $38 \mathrm{~dB}$. For P-waves, the attenuation of vertical vibration is negligible for frequencies below $a_{0} \approx 1$ (below $64 \mathrm{~Hz}$ for the $0.5 \mathrm{~m}$ thick slab considered in Section 5.1) but then increases steadily with frequency. For horizontal vibration, the low-frequency attenuation may be as high as $20 \mathrm{~dB}$ or more, but this depends strongly on the wave speed ratio and incidence angle; the minimum attenuation may be close to zero.

For SV-waves, the most significant observation is that the slab may amplify low-frequency vibration, particularly in the vertical direction, and by up to $10 \mathrm{~dB}$ depending on the wave speed ratio and incidence angle. To avoid any amplification, the slab must be sufficiently thick to ensure a minimum non-dimensional frequency of $a_{0} \approx 2$. This corresponds to a slab thickness of at least $2.5 \mathrm{~m}$, assuming the benchmark properties and that the minimum frequency of concern is $25 \mathrm{~Hz}$.

In general, for both P- and SV-waves, the vibration incidence angle is by far the most influential factor, causing variations in attenuation of $60 \mathrm{~dB}$ or more. This sensitivity presents a challenge to the designer, who may, at best, only estimate the incidence angle based on the approximate location of the source. 

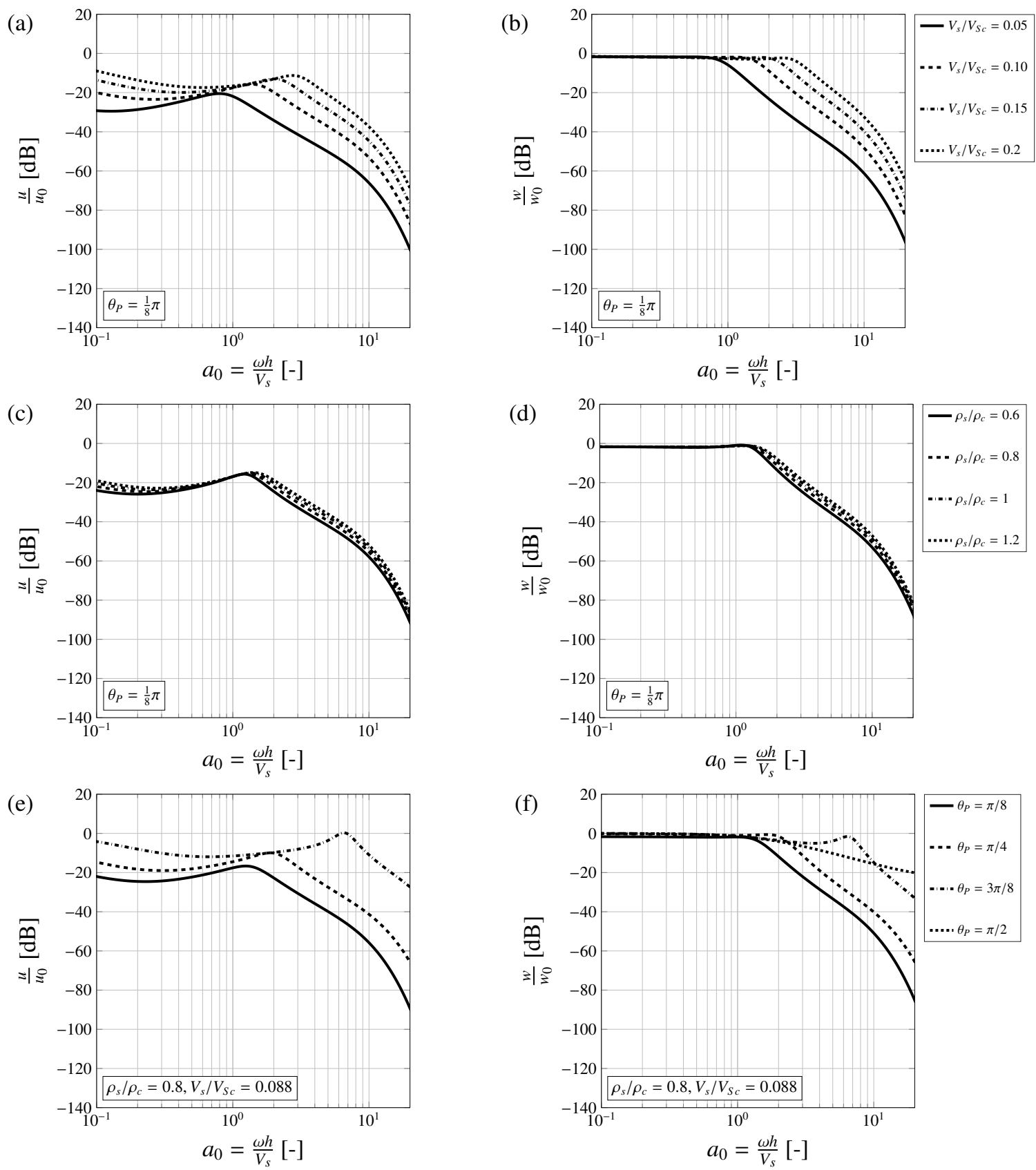

Figure 11: The horizontal (left) and vertical (right) displacement ratios at the free-surface of an infinitely large slab foundation subjected to an incident $\mathrm{P}$-wave at an angle $\theta_{P}=\pi / 8$, showing the influence of (a, b) the ratio $V_{s} / V_{S c}$, for $\rho_{s} / \rho_{c}=0.8$, and (c, d) the ratio $\rho_{s} / \rho_{c}$ for $V_{s} / V_{S c}=0.088$. (e, f) The influence of the incidence angle $\theta_{P}$ for $V_{s} / V_{S c}=0.088$ and $\rho_{s} / \rho_{c}=0.8$. 

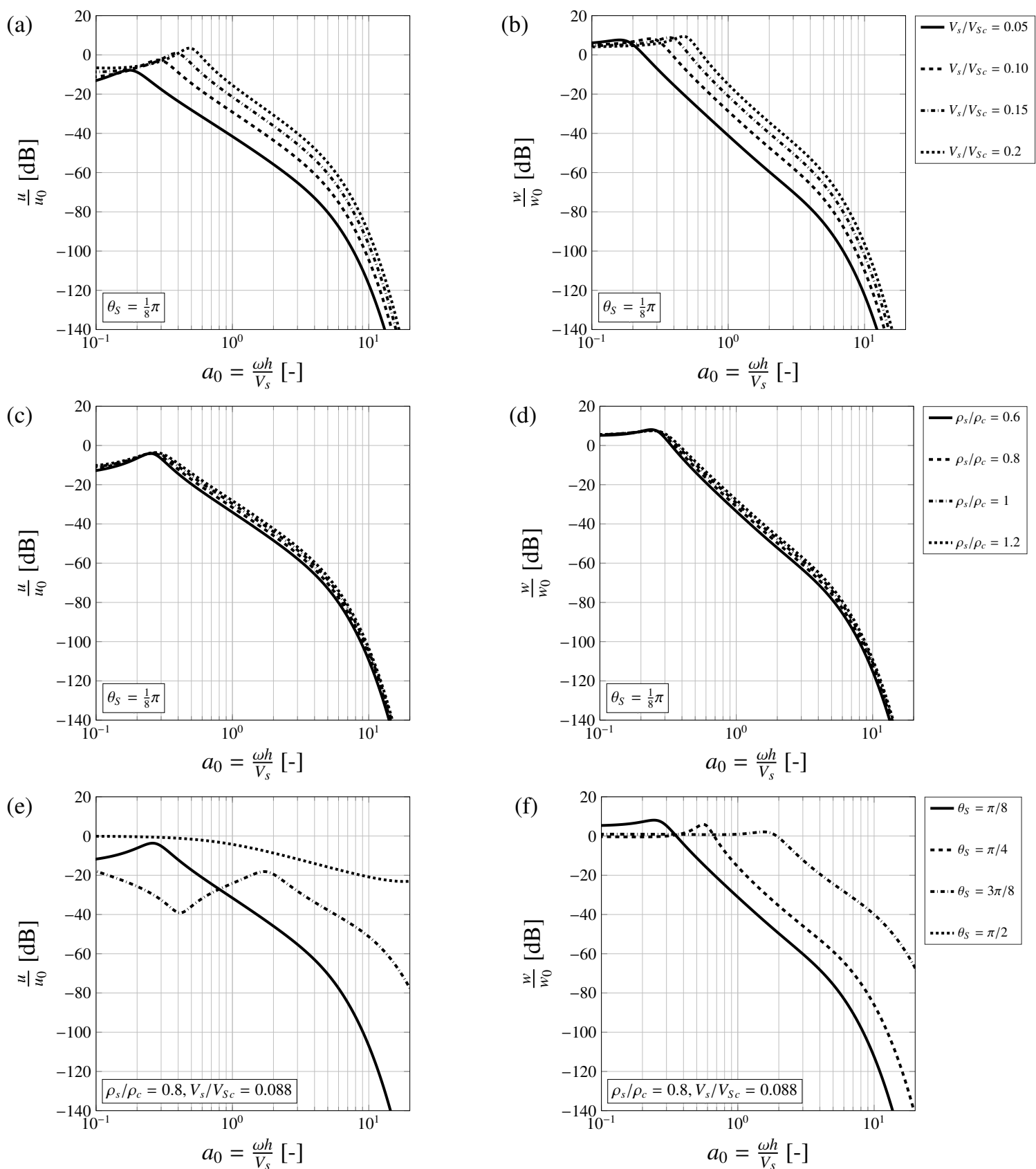

Figure 12: The horizontal (left) and vertical (right) displacement ratios at the free-surface of an infinitely large slab foundation subjected to an incident $\mathrm{SV}$-wave at an angle $\theta_{P}=\pi / 8$, showing the influence of (a, b) the ratio $V_{S} / V_{S c}$, for $\rho_{s} / \rho_{c}=0.8$, and $(\mathrm{c}, \mathrm{d})$ the ratio $\rho_{s} / \rho_{c}$ for $V_{S} / V_{S c}=0.088$. (e, f) The influence of the incidence angle $\theta_{S}$ for $V_{s} / V_{S c}=0.088$ and $\rho_{s} / \rho_{c}=0.8$. 

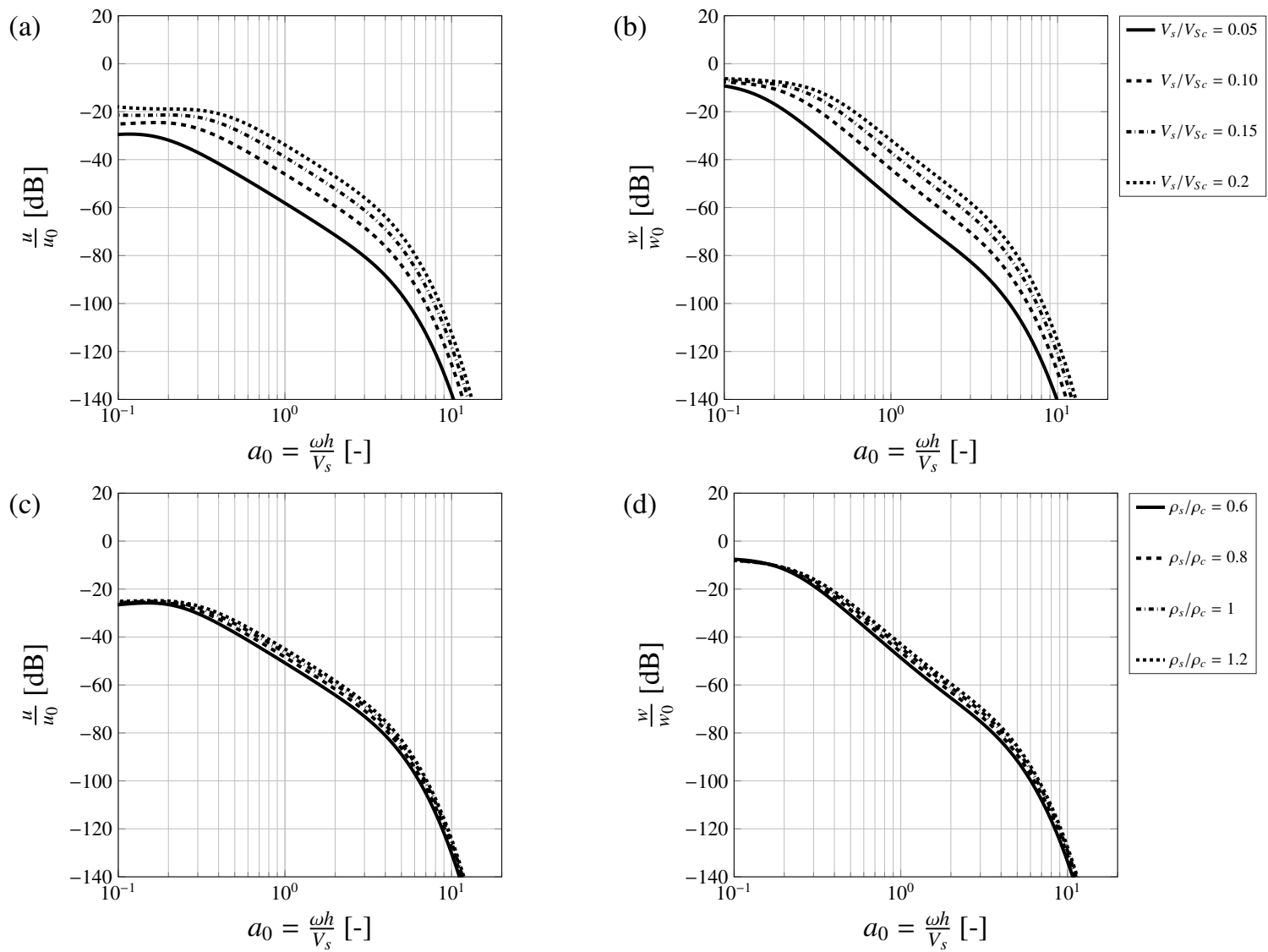

Figure 13: The horizontal (left) and vertical (right) displacement ratios at the free-surface of an infinitely large slab foundation subjected to an incident Rayleigh wave, showing the influence of (a,b) the ratio $V_{s} / V_{S c}$, for $\rho_{s} / \rho_{c}=0.8$, and (c, d) the ratio $\rho_{s} / \rho_{c}$ for $V_{s} / V_{S c}=0.088$.

\section{Conclusions}

This paper has considered the fundamental dynamic behaviour of a concrete slab foundation excited by using the corresponding dynamic stiffness matrices in a wave-based approach, the response of the slab to incident Rayleigh, P- and SV-waves has been explored. Having referred to previous work that assumes plate-like behaviour, it is clear that thin-plate theory alone is insufficient for modelling the effect of a slab foundation on an existing ground vibration field over the full frequency range of interest. Furthermore, models that assume a relaxed boundary condition at the soil-slab interface have been found to underestimate the attenuation provided by the slab by at least $10 \mathrm{~dB}$ in the case of Rayleigh wave excitation. 
The study has illustrated the importance of the coincidence phenomenon, in which the free-flexural wavelength of the slab coincides with the horizontal wavelength of the incident wave-field. For frequencies above coincidence, considerable attenuation can be achieved, but this depends on a number of dimensionless groups, as illustrated in a series of summary design plots. The least significant of these is the soil-slab density ratio, which, for all three wave types, has only a weak influence on the level of attenuation provided. In contrast, the relative stiffness of the slab (expressed as a ratio of wave speeds) has a strong influence. For a given site, the controlling parameter available to the foundation designer is the slab thickness. In the case of Rayleigh excitation, the design plots summarise concisely the behaviour of the slab, indicating that at least $20 \mathrm{~dB}$ of attenuation might reasonably be assumed for a typical, $0.5 \mathrm{~m}$ thick slab (considerably more at high frequencies). For P-and SV-waves, the situation is more complex because the coincidence frequencies associated with these wave types are always higher than that of the Rayleigh wave, which shifts the region of strong attenuation to relatively high frequencies. The overall attenuation therefore depends strongly on the slab stiffness and, importantly, the incidence angle of the vibration.

A fundamental assumption of this study is that the ground may be represented by a homogeneous halfspace, but this is often not the case due to soil layering. Layering introduces additional wave reflections and mode conversions, and this is likely to reduce the attenuation provided by the slab. The extent to which this is the case remains the subject of future research.

\section{Acknowledgements}

The authors gratefully acknowledge the support of Prof. Geert Degrande and colleagues in KU Leuven, who provided the Elastodynamics Toolbox for this work, together with the financial support of WSP and EPSRC (RG75686) for the studentship of the first author.

\section{References}

[1] H. Saurenman, J. Phillips, In-service tests of the effectiveness of vibration control measures on the BART rail transit system, J. Sound Vib. 293 (2006) 888-900.

[2] J. P. Talbot, Lift-over crossings as a solution to tram-generated ground-borne vibration and re-radiated noise, Proc. Inst. Mech. Eng. Part F J. Rail Rapid Transit 228 (2014) 878-886.

[3] P. Coulier, V. Cuéllar, G. Degrande, G. Lombaert, Experimental and numerical evaluation of the effectiveness of a stiff wave barrier in the soil, Soil Dyn. Earthq. Eng. 77 (2015) 238-253.

[4] D. J. Thompson, J. Jiang, M. G. R. Toward, M. F. M. Hussein, A. Dijckmans, P. Coulier, G. Degrande, G. Lombaert, Mitigation of railway-induced vibration by using subgrade stiffening, Soil Dyn. Earthq. Eng. 79 (2015) 89-103. 
[5] J. P. Talbot, Base-isolated buildings: towards performance-based design, Proc. Inst. Civ. Eng. - Struct. Build. 169 (2016) $574-582$.

[6] B. R. Barben, L. M. Hanagan, Investigation of a slab on grade supporting sensitive equipment, in: F. N. Catbas (Ed.), Mech. Biol. Syst. Mater. - Proc. 2013 Annu. Conf. Exp. Appl. Mech., Conference Proceedings of the Society for Experimental Mechanics Series, Springer New York LLC, Orlando, 2014, pp. 53-60.

[7] E. Kausel, Early history of soil-structure interaction, Soil Dyn. Earthq. Eng. 30 (2010) 822-832.

[8] H. L. Wong, J. E. Luco, Dynamic response of rectangular foundations to obliquely incident seismic waves, Earthq. Eng. Struct. Dyn. 6 (1978) 3-16.

[9] J. Qian, D. E. Beskos, Harmonic wave response of two 3-D rigid surface foundations, Soil Dyn. Earthq. Eng. 15 (1996) 95-110.

[10] M. Iguchi, J. E. Luco, Dynamic response of flexible rectangular foundations on an elastic half-space, Earthq. Eng. Struct. Dyn. 9 (1981) 239-249.

[11] D. J. Thompson, G. Kouroussis, E. Ntotsios, Modelling, simulation and evaluation of ground vibration caused by rail vehicles, Veh. Syst. Dyn. 0 (2019) 1-48.

[12] L. Auersch, Response to harmonic wave excitation of finite or infinite elastic plates on a homogeneous or layered half-space, Comput. Geotech. 51 (2013) 50-59.

[13] M. Schevenels, G. Degrande, S. François, EDT: An ElastoDynamics Toolbox for MATLAB, in: Proc. Inaug. Int. Conf. Eng. Mech. Inst., Minneapolis, U.S.A, pp. 82-82.

[14] M. Schevenels, S. François, G. Degrande, EDT: An ElastoDynamics Toolbox for MATLAB, Comput. Geosci. 35 (2009) $1752-1754$

[15] The Mathworks Inc., MATLAB 2018b, Natick, Massachusetts, United States, 2018.

[16] D. Connolly, G. Kouroussis, P. Woodward, P. Alves Costa, O. Verlinden, M. Forde, Field testing and analysis of high speed rail vibrations, Soil Dyn. Earthq. Eng. 67 (2014) 102-118.

[17] H. E. M. Hunt, Measurement and modelling of traffic-induced ground vibration, Ph.d. dissertation, University of Cambridge, 1988.

[18] E. Kausel, J. M. Roesset, Stiffness matrices for layered soils, Bull. Seismol. Soc. Am. 71 (1981) $1743-1761$.

[19] J. P. Talbot, H. E. M. Hunt, Isolation of Buildings from Rail-Tunnel Vibration: a Review, Build. Acoust. 10 (2003) 177-192.

[20] F. Fahy, P. Gardonio, Sound and Structural Vibration, Elsevier, 2006.

[21] K. Graff, Wave motion in elastic solids, Oxford: Clarendon, 1975.

[22] M. Schevenels, S. François, G. Degrande, EDT: elastodynamics toolbox for matlab, Technical Report July, Katholieke Universiteit Leuven, Leuven, 2010.

[23] W. Ewing, W. Jardetzky, F. Press, Elastic waves in layered media, McGraw-Hill, 1957. 
Table A.1: Benchmark Data

\begin{tabular}{|c|c|c|c|c|c|}
\hline \multirow[t]{6}{*}{ Soil } & Shear modulus & $G_{s}=8 \times 10^{7} \mathrm{~N} / \mathrm{m}^{2}$ & Slab & Young's Modulus & $E_{c}=3 \times 10^{10} \mathrm{~N} / \mathrm{m}^{2}$ \\
\hline & Shear wave speed & $V_{s}=200 \mathrm{~m} / \mathrm{s}$ & & Poisson ratio & $v_{c}=0.15$ \\
\hline & Poisson ratio & $v_{s}=1 / 3$ & & Mass density & $\rho_{c}=2500 \mathrm{~kg} / \mathrm{m}^{3}$ \\
\hline & Mass density & $\rho_{s}=2000 \mathrm{~kg} / \mathrm{m}^{3}$ & & Damping loss factor & $\eta_{c}=0.1$ \\
\hline & Damping loss factor & $\eta_{s}=0.1$ & & Width & $b=2,4,10,20, \underline{m} \mathrm{~m}$ \\
\hline & & & & Thickness & $t=0.7 \mathrm{~m}$ \\
\hline
\end{tabular}

\section{Appendix B. Displacement field $u_{0}$ and $w_{0}$ for incoming P- and SV-waves}

Consider an elastic and homogeneous half-space (Figure 1 1a) subjected to incident, plane P- or SV-waves at an angle $\theta_{P}$ or $\theta_{S}$ respectively. The wave equations for the P-SV problem can be written in terms of the potentials $\Phi$ and $\mathrm{H}_{y}[21,23]$ :

$$
\nabla^{2} \Phi=\frac{1}{V_{P}^{2}} \frac{\partial^{2} \Phi}{\partial t^{2}} ; \quad \nabla^{2} \mathrm{H}_{y}=\frac{1}{V_{S}^{2}} \frac{\partial^{2} \mathrm{H}_{y}}{\partial t^{2}}
$$

The solution can be found in terms of incoming and outgoing waves of amplitude $A_{1}, B_{1}$ and $A_{2}, B_{2}$ respectively, with reference to the potentials $\Phi$ and $\mathrm{H}_{y}$ :

$$
\Phi(x, z, t)=A_{1} \exp \left\{i\left(\omega t-k_{P} \cos \theta_{P} x+k_{P} \sin \theta_{P} z\right)\right\}+A_{2} \exp \left\{i\left(\omega t-k_{P} \cos \theta_{P} x-k_{P} \sin \theta_{P} z\right)\right\}
$$

$$
\mathrm{H}_{y}(x, z, t)=B_{1} \exp \left\{i\left(\omega t-k_{S} \cos \theta_{S} x+k_{S} \sin \theta_{S} z\right)\right\}+B_{2} \exp \left\{i\left(\omega t-k_{S} \cos \theta_{S} x-k_{S} \sin \theta_{S} z\right)\right\}
$$

The case of an incident $\mathrm{P}$ - or SV-wave can be explored by considering a known amplitude, $A_{1}$ or $B_{1}$, and imposing the stress-free condition at the free surface of the half-space to obtain the amplitudes $A_{2}$ and $B_{2}$ of the reflected waves:

\section{Case of incident P-wave}

$$
\frac{A_{2}}{A_{1}}=\frac{\sin 2 \theta_{P} \sin 2 \theta_{S}-\gamma^{2} \cos ^{2} 2 \theta_{S}}{\sin 2 \theta_{P} \sin 2 \theta_{S}+\gamma^{2} \cos ^{2} 2 \theta_{S}}
$$$$
\frac{B_{2}}{A_{1}}=\frac{2 \sin 2 \theta_{P} \cos 2 \theta_{S}}{\sin 2 \theta_{P} \sin 2 \theta_{S}+\gamma^{2} \cos ^{2} 2 \theta_{S}}
$$

\section{Case of incident SV-wave}

$$
\frac{B_{2}}{B_{1}}=\frac{\sin 2 \theta_{P} \sin 2 \theta_{S}-\gamma^{2} \cos ^{2} 2 \theta_{S}}{\sin 2 \theta_{P} \sin 2 \theta_{S}+\gamma^{2} \cos ^{2} 2 \theta_{S}}
$$$$
\frac{A_{2}}{B_{1}}=-\frac{\gamma^{2} \sin 4 \theta_{S}}{\sin 2 \theta_{P} \sin 2 \theta_{S}+\gamma^{2} \cos ^{2} 2 \theta_{S}}
$$ 

where $\gamma=V_{P} / V_{S}$ is the ratio between the pressure and shear wave speeds in the half-space. Finally, the 431 displacement amplitude $\mathbf{u}_{0}=\left[u_{0} w_{0}\right]$ at the free surface of the half-space can be retrieved from the potentials as:

\section{Case of incident P-wave}

$\frac{u_{0}}{-i k_{P} A_{1}}=\cos \theta_{P}\left(1+\frac{A_{2}}{A_{1}}\right)-\gamma \sin \theta_{S} \frac{B_{2}}{A_{1}}$

${ }_{432} \frac{w_{0}}{-i k_{P} A_{1}}=\sin \theta_{P}\left(\frac{A_{2}}{A_{1}}-1\right)+\cos \theta_{P} \frac{B_{2}}{A_{1}}$

\section{Case of incident SV-wave}

(B.7) $\frac{u_{0}}{-i k_{P} B_{1}}=\cos \theta_{P} \frac{A_{2}}{B_{1}}+\gamma \sin \theta_{S}\left(1-\frac{B_{2}}{B_{1}}\right)$

(B.8)

$$
\frac{w_{0}}{-i k_{P} B_{1}}=\sin \theta_{P} \frac{A_{2}}{B_{1}}+\cos \theta_{P}\left(1+\frac{B_{2}}{B_{1}}\right)
$$

